\title{
A rate-dependent Hosford-Coulomb model for predicting ductile fracture at high strain rates
}

\author{
Stephane J. Marcadet ${ }^{2}$, Christian C. Roth ${ }^{1,2}$, Borja Erice ${ }^{1}$, and Dirk Mohr ${ }^{1,2,3}$ \\ ${ }^{1}$ Solid Mechanics Laboratory (CNRS-UMR 7649), Department of Mechanics, École Polytechnique, Palaiseau, France \\ 2 Impact and Crashworthiness Lab, Department of Mechanical Engineering, Massachusetts Institute of Technology, \\ Cambridge MA, USA \\ ${ }^{3}$ Department of Mechanical and Process Engineering, ETH Zurich, Switzerland
}

\begin{abstract}
The Hosford-Coulomb model incorporates the important effect of the Lode angle parameter in addition to the stress triaxiality to predict the initiation of ductile fracture. A strain-rate dependent extension of the Hosford-Coulomb model is presented to describe the results from low, intermediate and high strain rate fracture experiments on advanced high strength steels (DP590 and TRIP780). The model predictions agree well with the experimental observation of an increase in ductility as function of strain rate for stress states ranging from uniaxial to equi-biaxial tension.
\end{abstract}

\section{Introduction}

Recent research on ductile fracture has clearly demonstrated that the effect of the Lode parameter is as important as that of the stress triaxiality. For example, the drastic loss in ductility of metals when changing the stress state from equi-biaxial tension (triaxiality of $2 / 3$ ) to plane strain tension (triaxiality of 0.58 ) cannot be explained using conventional stress triaxiality dependent models such as the Johnson-Cook failure model. However, Lode angle dependent theories such as the Hosford-Coulomb fracture initiation model (Mohr and Marcadet, 2015) can fully explain this phenomenon through the increased stability of plastic flow under axisymmetric stress states such as equibiaxial tension as compared to generalized shear stress states such as plane strain tension.

It is the objective of the present work to make use of these important recent findings on the stressstate dependency of ductile fracture to improve the predictions of ductile fracture initiation under dynamic loading conditions. For this, we present a rate-dependent version of the Hosford-Coulomb model. The proposed model may be seen as a Lode-angle dependent extension of the rate- and temperature dependent Johnson-Cook (1985) failure model. In most parts, this conference paper is compiled from extracts from the journal papers of Roth and Mohr (2014), Mohr and Marcadet (2015) and Gu and Mohr (2015). We first present the model formulation before demonstrating the model's ability to describe the experimental data from fracture experiments on advanced high strength steels that have been performed on a tension Hopkinson bar system.

\section{Theory}

\subsection{Definition of the Lode parameter and stress triaxiality dependency}

The stress state is described by the stress triaxiality and the Lode angle parameter. The stress triaxiality is defined as the ratio of the mean stress $\sigma_{m}$ and the von Mises stress $\bar{\sigma}$,

$$
\eta=\frac{\sigma_{m}}{\bar{\sigma}}
$$

It may be interpreted as a measure of the ratio of the first and second stress tensor invariants. The Lode angle parameter on the other hand measures the ratio of the third and second stress tensor invariants,

$$
\bar{\theta}=1-\frac{2}{\pi} \arccos \left[\frac{3 \sqrt{3}}{2} \frac{J_{3}}{\left(J_{2}\right)^{3 / 2}}\right] .
$$

According to the above definition, the Lode angle parameter varies between -1 (axisymmetric compression) and 1 (axisymmetric tension). Based on the modified Haigh-Westergaard coordinates, the ordered principal stresses $\left(\sigma_{i} \geq \sigma_{i i} \geq \sigma_{i i i}\right)$ may be constructed as

$$
\sigma_{i}=\bar{\sigma}\left(\eta+f_{i}\right)
$$

with the Lode angle parameter dependent trigonometric functions

$$
\begin{gathered}
f_{1}[\bar{\theta}]=\frac{2}{3} \cos \left[\frac{\pi}{6}(1-\bar{\theta})\right], \quad f_{2}[\bar{\theta}]=\frac{2}{3} \cos \left[\frac{\pi}{6}(3+\bar{\theta})\right], \\
f_{3}[\bar{\theta}]=-\frac{2}{3} \cos \left[\frac{\pi}{6}(1+\bar{\theta})\right] .
\end{gathered}
$$

\subsection{Stress-state dependent fracture initiation model}

Dunand and Mohr (2014) subjected a unit cell of a Levyvon Mises material with a central void of $1.2 \%$ porosity to combinations of shear and normal loading to determine the macroscopic equivalent plastic strain at the onset of localization as a function of the stress state. They

This is an Open Access article distributed under the terms of the Creative Commons Attribution License 4.0, which permits unrestricted use, distribution, and reproduction in any medium, provided the original work is properly cited. 


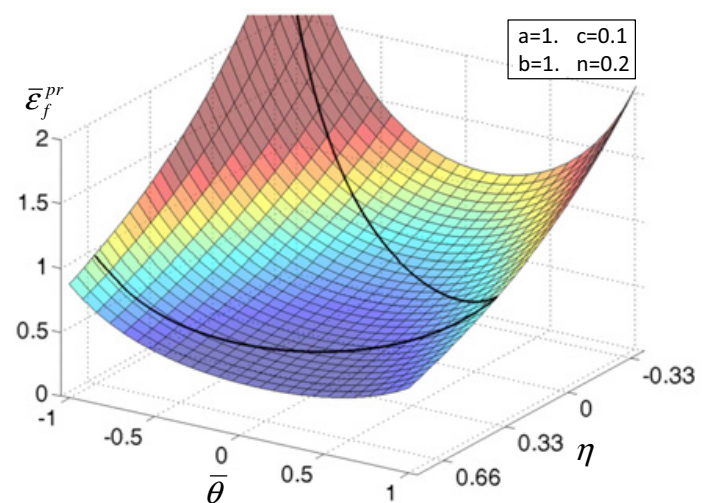

(a)

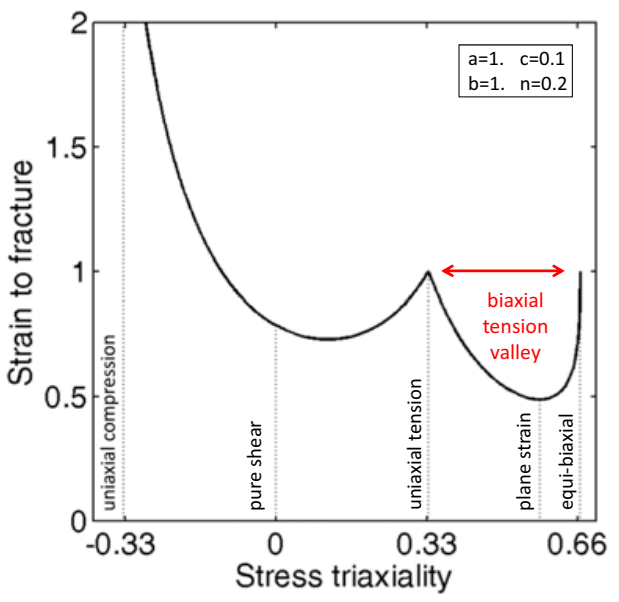

(b)

Figure 1. Hosford-Coulomb model for proportional loading: (a) strain to fracture as a function of the stress triaxiality and the Lode angle parameter; (b) fracture envelope for plane stress conditions.

performed this type of analysis for more than 160 different stress states for stress triaxialities ranging from 0 to 1 and Lode angle parameters ranging from -1 to 1 . Their results demonstrate that a Hosford-Coulomb criterion of the type

$$
\bar{\sigma}_{H F}+c\left(\sigma_{I}+\sigma_{I I I}\right)=\beta
$$

provides a good approximation of their simulation data, with the Hosford equivalent stress

$$
\bar{\sigma}_{H F}=\left\{\frac{1}{2}\left(\left(\sigma_{I}-\sigma_{I I}\right)^{a}+\left(\sigma_{I I}-\sigma_{I I I}\right)^{a}+\left(\sigma_{I}-\sigma_{I I I}\right)^{a}\right)\right\}^{\frac{1}{a}}
$$

and $1 \leq a \leq 2$ denoting the Hosford exponent. The onset of ductile fracture is considered to be imminent with the onset of localization. The above localization criterion (5) is therefore utilized to predict the onset of fracture under proportional loading. The results from ductile fracture experiments are typically presented in terms of the equivalent plastic strain, the stress triaxiality and the Lode angle parameter. We transform the localization criterion from the principal stress space to the mixed strain-stress

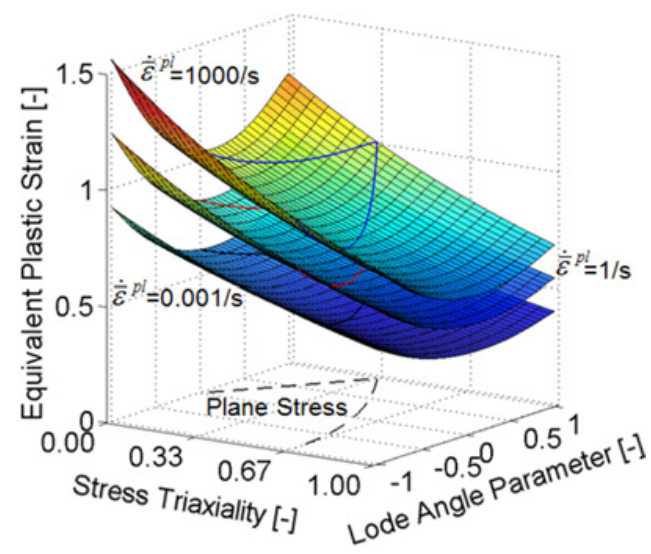

Figure 2. Illustration of the fracture surfaces (for proportional loading) for different strain rates.

space (for details, see Roth and Mohr, 2014),

$$
\begin{aligned}
& \bar{\varepsilon}_{f}^{p r}[\eta, \bar{\theta}]=b(1+c)^{\frac{1}{n}} \ldots \\
& \quad \ldots\left(\left\{\frac{1}{2}\left(\left(f_{1}-f_{2}\right)^{a}+\left(f_{2}-f_{3}\right)^{a}+\left(f_{1}-f_{3}\right)^{a}\right)\right\}^{\frac{1}{a}}\right. \\
& \left.\quad+c\left(2 \eta+f_{1}+f_{3}\right)\right)^{-\frac{1}{n}}
\end{aligned}
$$

Figure 1a shows a plot of the isotropic Hosford-Coulomb fracture initiation model for proportional loading. The strain to fracture is a monotonically decreasing function of the stress triaxiality and a convex non-symmetric function of the Lode angle parameter. The corresponding strain to fracture for plane stress conditions (see black curve in Fig. 1a) is shown as a function of the stress triaxiality only in Fig. 1b. Note that the apparent non-monotonic dependence on the stress triaxiality is deceiving because of the underlying Lode angle effect that is due to the functional relationship $\bar{\theta}=\bar{\theta}[\eta]$ for plane stress. The main model parameters are $\{a, b, c\}$. The Hosford exponent $a$ controls the effect of the Lode angle parameter, while the friction coefficient $c$ primarily controls the effect of the stress triaxiality on the strain to fracture. The model parameter $b$ is a multiplier controlling the overall magnitude of the strain to fracture; it is defined such that it is equal to the strain to fracture for uniaxial tension (which is the same as that for equi-biaxial tension). The parameter $n=0.1$ is a fixed transformation parameter. Note that we introduced the superscript "pr" to indicate that the above expressions are applied to predict the onset of fracture under proportional loading only. For nonproportional loading, the integral form

$$
\int_{0}^{\bar{\varepsilon}_{f}} \frac{d \bar{\varepsilon}_{p}}{\bar{\varepsilon}_{f}^{p r}[\eta, \bar{\theta}]}=1
$$

is used, where the expression for the strain to fracture for proportional loading serves as weighting function.

\subsection{Rate-dependent Hosford-Coulomb model}

In loose analogy with the Johnson and Cook (1985) fracture model, the effect of strain rate is incorporated into 


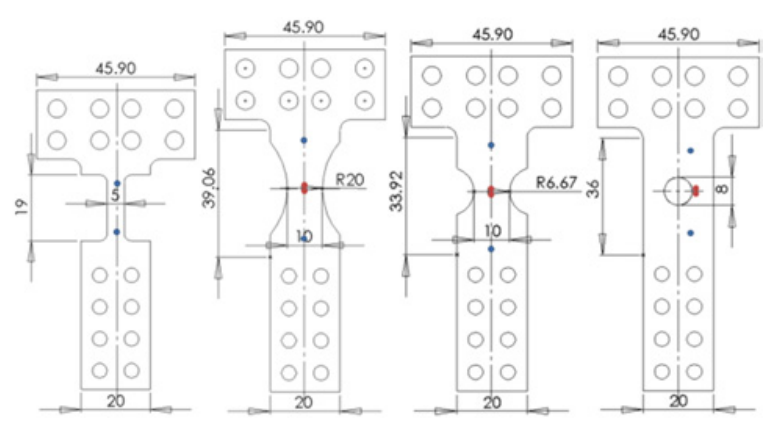

Figure 3. Specimen geometries for high strain rate testing.

the Hosford-Coulomb model through the coefficient $b$,

$$
b= \begin{cases}b_{0} & \text { for } \dot{\bar{\varepsilon}}_{p}<\dot{\varepsilon}_{0} \\ b_{0}\left(1+\gamma \ln \left[\frac{\dot{\bar{\varepsilon}}_{p}}{\dot{\varepsilon}_{0}}\right]\right) & \text { for } \dot{\bar{\varepsilon}}_{p} \geq \dot{\varepsilon}_{0}\end{cases}
$$

$b_{0}>0$ is the strain to fracture for uniaxial tension at low strain rates, while the model parameter $\gamma \geq 0$ controls the strain rate sensitivity of the fracture initiation model. Figure 2 illustrates the effect of the strain rate on the resulting "fracture surface" in the space of equivalent plastic strain, stress triaxiality and Lode angle parameter.

\section{Experimental results}

\subsection{Material, specimens and experimental procedures}

The tested materials are $1.4 \mathrm{~mm}$ thick DP590 steel and $1.4 \mathrm{~mm}$ thick TRIP780 steel. Uniaxial tension (UT), notched tension (NT) and central hole $(\mathrm{CH})$ tension specimens (Fig. 3) are extracted from these sheets. Prior to testing, a random speckle pattern is applied to the specimens. The relative displacement of two points on the respective upper and lower specimen shoulders (see blue dots in Fig. 3) is measured in all experiments by means of digital image correlation (VIC2D, Correlated Solutions). The distance between these points is equal to $l_{0}=17.5 \mathrm{~mm}$ for the UT-specimens, and $l_{0}=30 \mathrm{~mm}$ for the NT and $\mathrm{CH}$ specimens. The experiments at low and intermediate strain rates are carried out on a MTS hydraulic testing machine with a $250 \mathrm{kN}$ load cell and custom-made high pressure clamps.

\subsection{Experimental results}

The effect of the strain rate on the engineering stress-strain curve for DP590 steel for uniaxial tension along the rolling direction is illustrated by Fig. 4. Observe that the material's yield stress is about $17 \%$ higher in the intermediate strain rate experiment than in the low strain rate experiment. It is also worth noting that the elongation to fracture (engineering strain at the onset of fracture as evaluated for an extensometer length of $17.5 \mathrm{~mm}$ ) is significantly higher in the low strain rate experiment than in the intermediate and high strain rate experiments.

Figure 5 shows the force-displacement curves experiments for three different speeds of loading which are also qualitatively representative for the NT6 and $\mathrm{CH}$

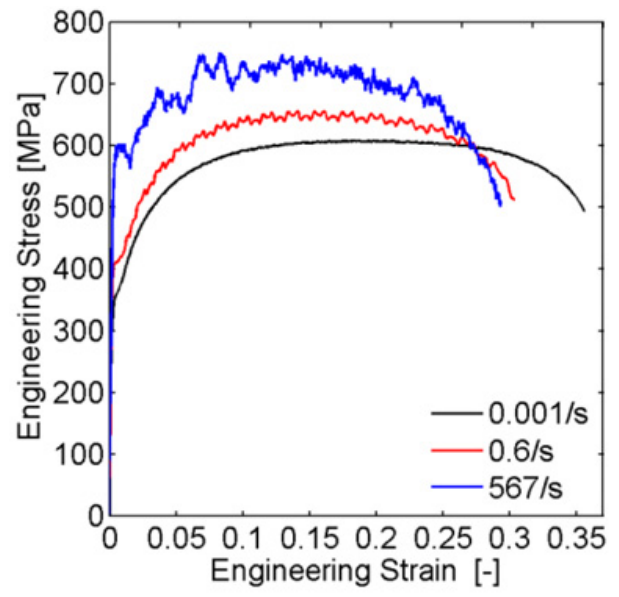

Figure 4. Stress-strain response for DP590.

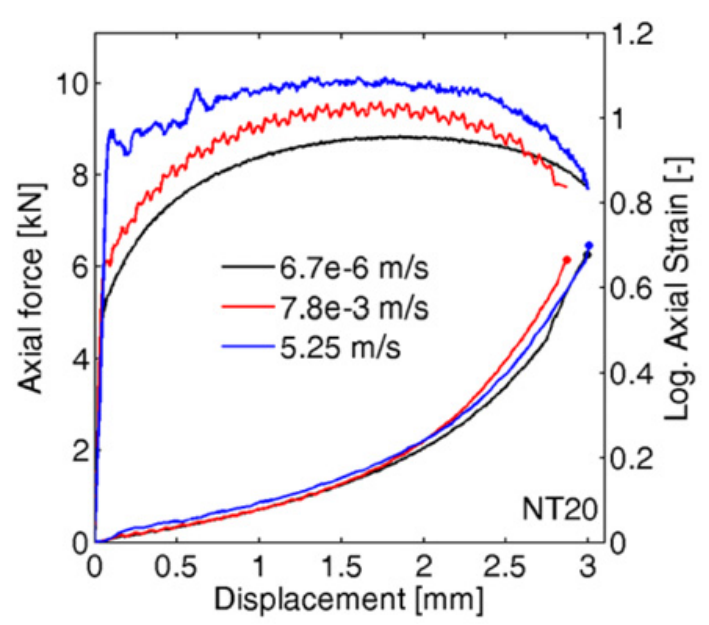

Figure 5. Force-displacement curve for notched tension (NT20).

experiments; All experiments show a force maximum prior to the onset of fracture, which increases as a function of the loading speed. The shapes of the force-displacement curves for low and intermediate strain rates are similar up to the force maximum, i.e. a dynamic increase factor could be used to describe the effect of the loading speed.

The shape of the curve for the fast loading speed is different, i.e. it exhibits a significantly higher force level at the beginning of the experiment followed by a less pronounced hardening regime. Note that after the force maximum, the decrease in force is higher the faster the loading speed. However, it is remarkable to see that for all three specimen types, the displacement to fracture appears to be almost independent of the loading speed. To gain further insight into the local deformation fields, we also report the strain determined from a $1 \mathrm{~mm}$ long virtual extensometer on the specimen surface (see secondary axis in Fig. 4). The evolution of the local strain as a function of the axial displacement is very similar irrespective of the speed of loading. In particular, only small variations are observed at the onset of fracture. 

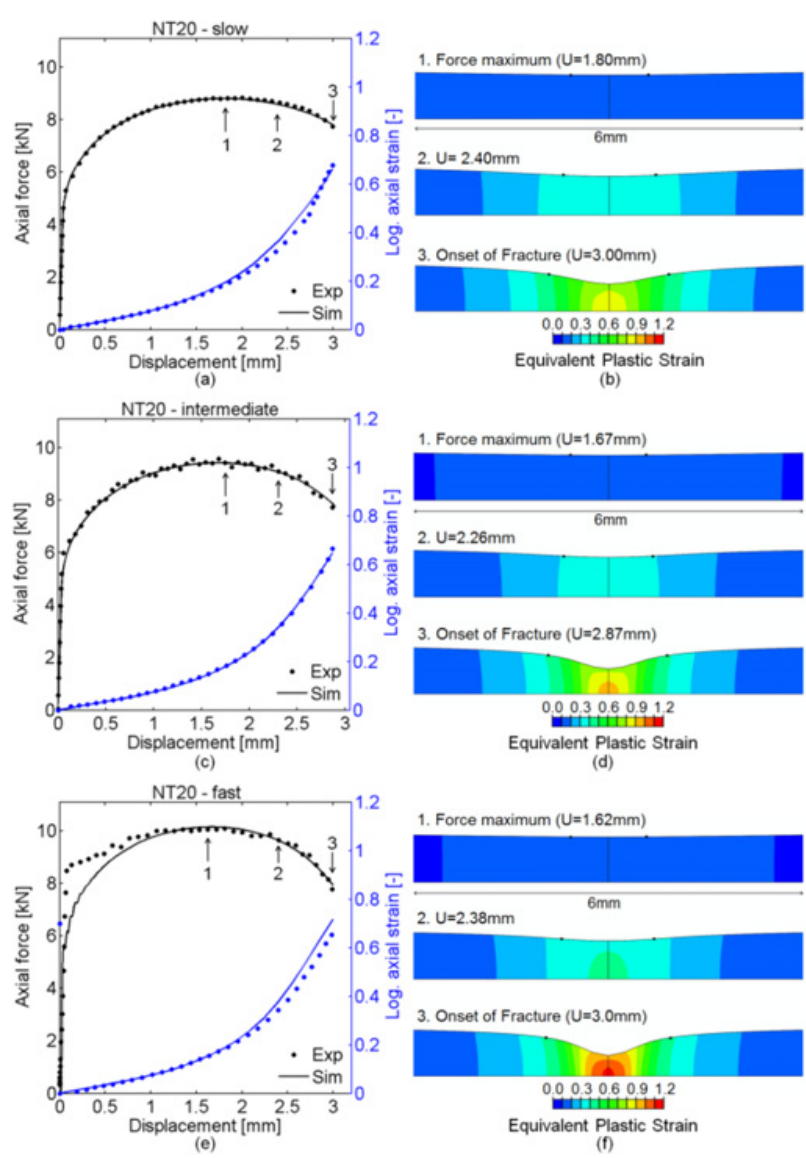

Figure 6. Force-displacement curve for notched tension NT20 (left column) and longitudinal cross-sectional views (right column).

\section{Model calibration and validation}

Numerical simulations are performed of all experiments using the finite element software Abaqus/explicit along with a strain rate and temperature-dependent plasticity model (Roth and Mohr, 2014). An example for the comparison of measured (solid dots) and computed (solid lines) force-displacement curves is shown in Fig. 6. The final result from each simulation is the so-called loading path to fracture, i.e. the evolution of the equivalent plastic strain $\bar{\varepsilon}_{p}$ as a function of the strain rate $\dot{\bar{\varepsilon}}_{p}$, the temperature $T$, the stress triaxiality $\eta$ and the Lode angle parameter $\bar{\theta}$. It is assumed that fracture initiates in the simulation at the integration point with the highest equivalent plastic strain at the instant of specimen fracture in the experiment. For the NT20 and NT6 specimens, this point is positioned at the very center of the specimen, i.e. at the intersection point of all planes of specimen symmetry. In the $\mathrm{CH}$ specimens, this point is positioned on the specimen mid-plane at a distance of about $0.5 \mathrm{~mm}$ from the central hole boundary.

Figure 7 shows the loading paths to fracture in terms of the equivalent plastic strain and the stress triaxiality for NT20 specimens. The cross symbols on the loading paths indicate the point at which the force maximum is reached. Irrespective of the specimen type (NT20, NT6, $\mathrm{CH})$, the stress state is independent of the strain rate and remains approximately constant up to the force maximum. Thereafter, the stress triaxiality increases while the Lode

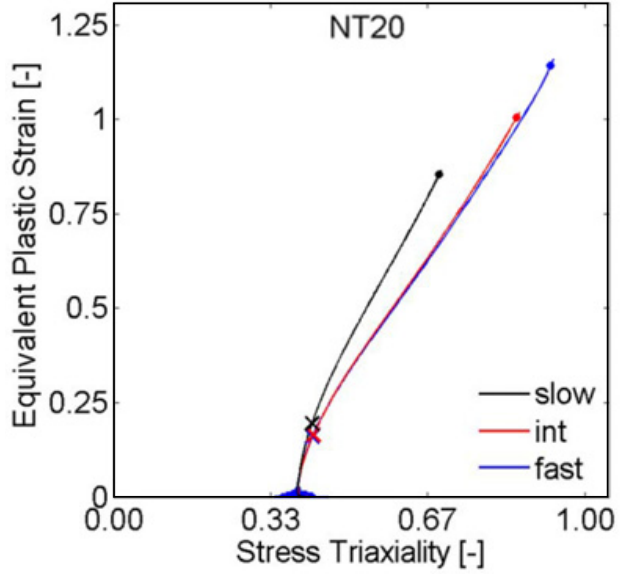

Figure 7. Loading paths to fracture for notched tension (DP590 steel).

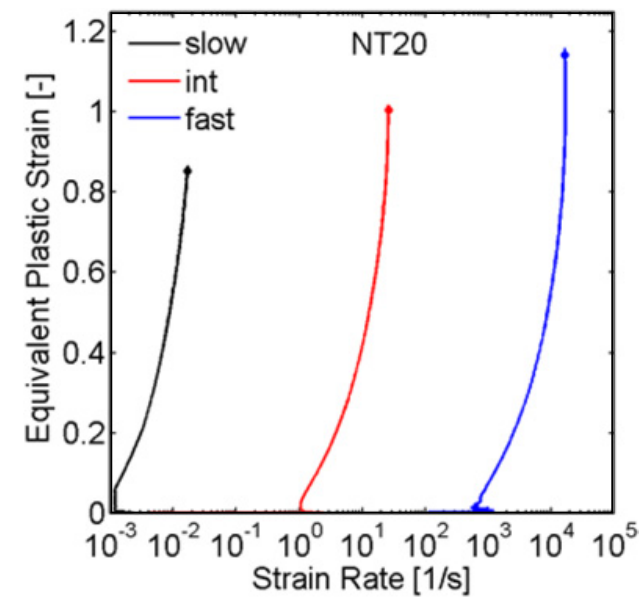

Figure 8. Force-displacement curve for notched tension (NT20).

angle parameter decreases. This is attributed to the builtup of an out-of-plane stress during through-thickness necking. Due to the absence of thermal softening for slow loading, necking is less pronounced for this case than for intermediate and fast loading. The longitudinal crosssectional views of the specimens (e.g. right columns in Fig. 6) show the strain distribution throughout the necking phase. Up to the force maximum, plane stress conditions prevail and the strain distribution is approximately uniform along the thickness direction. Throughout necking, the deformation localizes within a small zone along the longitudinal axis and through-thickness gradients built up.

The comparison of the cross-sectional views at the onset of fracture for different loading velocities clearly shows that the localization severity increases the higher the speed of loading. The corresponding plots of the equivalent plastic strain versus the strain rate (Fig. 8) show that the strain rate increases by at least one order of magnitude after the onset of necking. For example, the pre-necking strain rate in the fast NT20 experiment is about $10^{3} / \mathrm{s}$, while a strain rate of about $10^{4} / \mathrm{s}$ is reached at the onset of fracture.

The analysis of the loading paths to fracture for different stress states (NT20, NT6, CH) and different materials (DP590, TRIP780) supports the assumption made in the rate-dependent Hosford-Coulomb model: the 
strain to fracture increases as a function of the loading speed. The solid dots in Fig. 8 are the predictions of the instant of fracture initiation by the Hosford-Coulomb model; these dots almost coincide with the end of the solid curves which corresponds to the instant of fracture initiation observed in the experiments.

\section{Conclusions}

A rate-dependent Hosford-Coulomb fracture initiation model is presented and used to predict the onset of fracture in static and dynamic experiments on advanced high strength steel (DP590, TRIP780) for different stress states and different loading velocities. The model may be seen as an extension of the Johnson-Cook failure model; however, it accounts not only for the effect of strain rate and stress triaxiality, but it also incorporates the important effect of the Lode parameter on fracture initiation. The experimental results demonstrate that for biaxial tension, the strain to fracture increases as a function of the loading velocity. Due to the adiabatic heating during the dynamic experiments, the effects of strain rate and temperature could not be separated in the present analysis.

$\mathrm{CR}$ and $\mathrm{BE}$ are grateful for the partial financial support through the French National Research Agency (Grant ANR-11-BS09-0008, LOTERIE). The authors are also grateful for the support of the MIT Industrial Fracture Consortium.

\section{References}

[1] C.C. Roth and D. Mohr, Effect of Strain Rate on Ductile Fracture Initiation in Advanced High Strength Steel Sheets: Experiments and Modeling. International Journal of Plasticity 56 (2014) 19-44.

[2] D. Mohr and S. J. Marcadet, Micromechanicallymotivated Phenomenological Hosford-Coulomb Model for Predicting Ductile Fracture Initiation at Low Stress Triaxialites, Int. J. Solids Struct (2015), in press.

[3] G. Gu and D. Mohr, Anisotropic Hosford-Coulomb Fracture Initiation Model: Theory and Application (2015), under review.

[4] Dunand M, Mohr D (2014). Effect of Lode parameter on plastic flow localization after proportional loading at low stress triaxialities, J. Mech. Phys. Solids 66, 133-153.

[5] Dunand M, Gary G, Mohr D (2013), Load-Inversion Device for the High Strain Rate Tensile Testing of Sheet Materials with Hopkinson Pressure Bars, Exp. Mech. 53, 1177-1188

[6] G.R. Johnson and W.H. Cook, Fracture characteristics of three metals subjected to various strains, strain rates, temperatures and pressures. Engineering Fracture Mechanics (1985), 21, p. 31-48. 